\title{
Lipopolysaccharide-Induced Osteoclastogenesis from Mononuclear Precursors: A Mechanism for Osteolysis in Chronic Otitis
}

\author{
Robert Nason, ${ }^{1}$ Jae Y. Jung, ${ }^{1}$ and Richard A. Chole ${ }^{1,2,3}$ \\ ${ }^{1}$ Department of Otolaryngology/Head and Neck Surgery, Washington University School of Medicine, St. Louis, MO, USA \\ ${ }^{2}$ Molecular Pharmacology, Washington University School of Medicine, St. Louis, MO, USA \\ ${ }^{3}$ Central Institute for the Deaf, Washington University School of Medicine, St. Louis, MO, USA
}

Received: 29 May 2008; Accepted: 3 December 2008; Online publication: 15 January 2009

\begin{abstract}
Osteoclasts are the only cells capable of carrying out bone resorption and therefore are responsible for the osteolysis seen in infectious diseases such as chronic otitis media and infected cholesteatoma. Pseudomonas aeruginosa is the most common organism isolated from these infectious middle ear diseases. In this study, we examined the mechanisms by which $P$. aeruginosa lipopolysaccharide (LPS) stimulates osteoclastogenesis directly from mononuclear osteoclast precursor cells. Osteoclast precursors demonstrated robust, bone-resorbing osteoclast formation when stimulated by $P$. aeruginosa LPS only if previously primed with permissive, sub-osteoclastogenic doses of receptor activator of NF- $\kappa B$ ligand (RANKL), suggesting that LPS is osteoclastogenic only during a specific developmental window. Numerous LPS-elicited cytokines were found to be released by osteoclast precursors undergoing $P$. aeruginosa LPS-mediated osteoclast formation. Two lines of evidence suggest that several cytokines promote Oc formation in an autocrine/paracrine manner. First, inhibition of several cytokine pathways including TNF- $\alpha$, IL-1, and IL6 block the osteoclastogenesis induced by LPS. Secondly, increased expression of the receptors for TNF- $\alpha$ and IL-1 was demonstrated by real-time quantitative polymerase chain reaction. Such a mechanism has not previously been established and dem-
\end{abstract}

Correspondence to: Richard A. Chole · Department of Otolaryngology/ Head and Neck Surgery - Washington University School of Medicine · St. Louis, MO, USA. Telephone: +1-314-7474388; fax: +1-314-7471004; email: rchole@wustl.edu onstrates the ability of osteoclast precursors to autonomously facilitate bone destruction.

Keywords: chronic otitis media, cholesteatoma, inflammatory osteolysis, RANKL

\section{INTRODUCTION}

Bone is a dynamic organ constantly being remodeled to achieve both calcium homeostasis and structural integrity-matrix synthesis is carried out by osteoblasts (Obs), while resorption is performed exclusively by osteoclasts (Ocs). Under normal physiologic conditions, these activities are so closely balanced that as much as $10 \%$ of total bone content is replaced per year in an adult human (Alliston and Derynck 2002). Otologic conditions that disrupt this balance between bone formation and resorption are chronic otitis media (COM) and infected cholesteatomas. In these conditions, infection leads to inflammation adjacent to bone, resulting in increased Oc activation and accelerated bone erosion. Bones affected include the ossicles, labyrinth, and temporal bone. Pathologic destruction of these structures can lead to hearing loss, vertigo, facial paresis, and possible intracranial invasion.

Ocs, the only cells capable of carrying out bone resorption, are multinucleated cells that form by fusion of bone marrow-derived mononuclear precursors (Udagawa et al. 1990). It has been well established that Oc formation from monocyte Oc precursors 
(pOcs) is regulated by two essential cytokines: receptor activator of NF-kB ligand (RANKL) and macrophage colony-stimulating factor (M-CSF; Felix et al. 1990; Lacey et al. 1998). Under physiologic conditions, Ocs are generated when pOcs are in direct contact with RANKL-generating supporting cells (e.g., Obs, stromal cells, and their precursors). These supporting cells secrete M-CSF and carry membranebound RANKL to initiate osteoclastogenesis by engaging the $c$-Fms and RANK receptors on pOcs, respectively. In pathologic conditions like COM and infected cholesteatoma, however, RANKL becomes a soluble factor produced in large concentrations by lymphocytes (Kong et al. 1999).

Although the pathogenesis is poorly understood, bacteria play a role in inciting the bone destruction seen in COM and infected cholesteatomas. The most common organism cultured from COM and infected cholesteatomas is the Gram-negative bacillus, Pseudomonas aeruginosa (Fliss et al. 1991; Nakagawa et al. 2004). One of the most important virulence factors from Gram-negative bacteria is lipopolysaccharide (LPS), the main antigenic component of the bacterial cell wall. LPS was recently shown to be present at higher concentrations in cholesteatoma samples from patients with clinical evidence of bone resorption, highlighting the contributory role that LPS plays in propagating pathologic bone destruction (Peek et al. 2003). Due to the prevalence of $P$. aeruginosa in chronic middle ear diseases, we investigated the role of $P$. aeruginosa LPS in osteoclastogenesis and previously demonstrated the osteoclastogenic potential of P. aeruginosa LPS in co-culture systems (Zhuang et al. 2007). No osteoclastogenesis was seen, however, when $P$. aeruginosa LPS was added to purified pOc monocultures (Zhuang et al. 2007). One likely explanation is that pOcs may respond to LPS in a pro-osteoclastogenic manner only during a specific developmental window (Lam et al. 2000; Zou and Bar-Shavit 2002). In order to delineate this process, we designed a series of experiments to assess the effect of sub-osteoclastogenic RANKL (10 ng/ml) pre-exposure on pOcs in the process of $P$. aeruginosa LPS-induced osteoclastogenesis.

The present study focused on whether $P$. aeruginosa LPS could directly stimulate pOcs to form bone-resorbing multinucleated Ocs without the need for supporting cells. To avoid the possibility of capturing indirect effects from non-pOc cells, our current study utilized monocultures of primary bone marrow pOcs. Studies with primary pOcs were verified with RAW 264.7 cells, a purified murine hematopoietic cell line. In this manner, we have identified autocrine/paracrine pro-osteoclastogenic mechanisms involving inflammatory cytokines elicited by $P$. aeruginosa LPS.

\section{MATERIALS AND METHODS}

\section{Animals}

Bone marrow from male, age-matched C57/BL6 mice was used in this study as a source of pOcs. Additionally, bone marrow from knockout mice was also used in this study. Knockout mice were on a C57/BL6 background and possessed targeted deletions of either IL-6 (IL-6 ${ }^{-/-}$; Kopf et al. 1994), TNF receptor 1 (TNFR1 $^{-/-}$; Peschon et al. 1998), TNF receptor 2 (TNFR2 $^{-/-}$; Erickson et al. 1994), both TNF receptors 1 and 2 (TNFR1/2 $2^{-/-}$; Peschon et al. 1998), or IL-1 receptor 1 (IL-1R1 $1^{-/-}$; Glaccum et al. 1997). All mice were obtained from Jackson Laboratories (Bar Harbor, ME, USA). Use of these vertebrate animals was approved by the Washington University Animal Studies Committee.

\section{Reagents}

$P$ aeruginosa LPS (serotype 10), recombinant murine M-CSF, media, and penicillin-streptomycin media supplements were purchased from Sigma. Fetal calf serum was purchased from Invitrogen. Murine IL-1 receptor antagonist (IL-1ra) was purchased from R\&D Systems. Recombinant glutathione $S$-transferase (GST)-RANKL was generated and purified in our laboratory from a plasmid provided by Dr. Steven Teitelbaum (Washington University School of Medicine, St. Louis, MO, USA). Purified RANKL protein was tested for LPS contamination by limulus amoebocyte lysate assay (Cambrex Bio Services) and found to contain negligible LPS.

\section{Osteoclast precursor culturing system}

Bone marrow monocytes were obtained from dissected femurs of 5- to 8-week-old male C57/BL6 mice. Cells were suspended in $\alpha$-minimal essential medium (Sigma) containing $1 \%$ penicillin-streptomycin, $10 \%$ fetal calf serum, and cultured overnight at $37^{\circ} \mathrm{C}$ in $5 \%$ $\mathrm{CO}_{2}$. Non-adherent cells were gently washed from the plate the following day, resuspended, and plated into 96-well culture plates at $0.5 \times 10^{6}$ cells $/ \mathrm{ml}$ in media further supplemented with recombinant murine $\mathrm{M}$ CSF $(10 \mathrm{ng} / \mathrm{ml})$ and a permissive dose of RANKL $(10 \mathrm{ng} / \mathrm{ml})$. Importantly, this permissive dose will not induce Oc formation in pOcs as determined by dose response experiments in our laboratory. These experiments have also demonstrated that RANKL at $100 \mathrm{ng} /$ $\mathrm{ml}$ is maximally osteoclastogenic (unpublished data). After plating into 96-well culture plates, pOc monocultures were exposed to a permissive or "priming" dose of RANKL (10 ng/ml) for either 0 , 24, 48, or $72 \mathrm{~h}$ prior to $P$. aeruginosa LPS exposure $(1 \mu \mathrm{g} / \mathrm{ml})$; 
once LPS was added, cultures were continued in media containing either $P$. aeruginosa LPS $(1 \mu \mathrm{g} / \mathrm{ml})$ and RANKL $(10 \mathrm{ng} / \mathrm{ml})$ or $P$. aeruginosa LPS $(1 \mu \mathrm{g} / \mathrm{ml})$ without RANKL. Total culture duration was 7 days from the time of initial plating since it required approximately $96 \mathrm{~h}$ to achieve maximal Oc formation. Media were replaced every other day.

In addition, RAW 264.7 cells (ATCC \#TIB-71, Rockville, MD, USA), a murine hematopoietic cell line, was used to corroborate findings from primary cells. This is because primary pOc preparations always possess a small fraction of non-pOc cell types and may also exist in varied states of differentiation (Zhuang et al. 2007). RAW 264.7 cells were plated into 96-well culture plates at $1 \times 10^{5}$ cells $/ \mathrm{ml}$ in Dulbecco's modified Eagle's medium (Sigma) containing 10\% fetal calf serum, $1 \%$ penicillin-streptomycin, and permissive RANKL $(10 \mathrm{ng} / \mathrm{ml})$. M-CSF was not used in RAW 264.7 cell cultures since M-CSF is constitutively expressed by these cells (Hsu et al. 1999). Upon plating, these cells were treated similarly to the primary pOcs as described above. Ocs formation from RAW cells was significantly faster than in primary pOcs secondary to their constitutive expression of MCSF.

At the end of experiments, cells were washed with phosphate-buffered saline, fixed with $4 \%$ paraformaldehyde, and stained for tartrate-resistant acid phosphatase (TRAP) using the manufacturer's recommended protocol (Sigma).

\section{Osteoclast quantitation}

Individual wells of a 96-well plate were imaged at room temperature using a digital camera (Sony DKC5000, Japan) coupled to an inverted microscope (Olympus IMT-2, Japan). The entirety of each well was captured with the $1 \times$ objective. TRAP-stained cells with three or more nuclei were counted as Ocs. Digital images of each well were manipulated using Adobe Photoshop (Adobe Systems Incorporated). Ocs in each image were manually replaced with masks, while all non-Oc image contents were cleared. Converted images of positively-selected Oc masks were further analyzed with the image analysis program, Image J (Rasband 1997-2007). Oc formation was assessed in Image J by quantifying two parameters: (1) area fraction, a measure of the total Oc surface area occupying each well and (2) the total number of Ocs formed in each well.

\section{Assessment of bone resorption}

For resorption studies, pOcs were plated directly onto either dentin slices or Osteologic ${ }^{\mathrm{TM}}$ hydroxyapatitecoated culture slides (Becton Dickinson) at $0.5 \times 10^{6}$ cells $/ \mathrm{ml}$. For these studies, cetacean dentin was cut into 500- $\mu \mathrm{m}$-thick slices using a Buehler Isomet low speed saw (Irvine, CA, USA). These slices were then sterilized in $70 \%$ ethanol prior to use. After plating, pOcs were primed for $72 \mathrm{~h}$ with RANKL at $10 \mathrm{ng} / \mathrm{ml}$ before being exposed to $P$. aeruginosa LPS $(1 \mu \mathrm{g} / \mathrm{ml})$. Media were replaced every other day. Experiments on Osteologic ${ }^{\mathrm{TM}}$ slides were carried out for 7 days, whereas dentin experiments were carried out for 10-15 days. After culturing, cells were removed from dentin slices by sonication. Cells were removed from Osteologic ${ }^{\mathrm{TM}}$ slides with a $5.25 \%$ sodium hypochlorite solution. Sonicated dentin slices were stained with a $1 \%$ toluidine blue $/ 1 \%$ sodium tetraborate solution, and resorption areas on both substrates were imaged using a digital camera (Sony DKC-5000, Japan) coupled to an inverted microscope (Olympus IMT-2, Japan).

\section{Cytokine profiling}

For cytokine analysis, Raybio ${ }^{\circledR}$ Mouse Inflammation Antibody Array I (Raybiotech, Norcross, GA, USA) was used following the manufacturer's protocol. Two membrane arrays were run independently for each culture condition. Conditioned cell culture supernatants from three different treatment groups were assayed for 40 inflammatory cytokines. These groups were: (1) untreated RAW 264.7 cells in serumcontaining DMEM media which served as a baseline control; (2) RAW 264.7 cells primed with RANKL (10 ng/ml) for $72 \mathrm{~h}$; and (3) RAW 264.7 cells primed with RANKL (10 $\mathrm{ng} / \mathrm{ml})$ for $72 \mathrm{~h}$ and then stimulated with $P$. aeruginosa LPS $(1 \mu \mathrm{g} / \mathrm{ml})$ and RANKL $(10 \mathrm{ng} / \mathrm{ml})$ for an additional $48 \mathrm{~h}$.

Chemiluminescent signal intensities were quantified with a Bio-Rad VersaDoc ${ }^{\mathrm{TM}}$ Imaging System (Bio-Rad). For analysis, Quantity One ${ }^{\mathrm{TM}}$ software (Bio-Rad) was used to determine the net optical density level for each array spot. This was calculated by subtracting the background optical level from the total raw optical density level. The average of both runs was then calculated for each cytokine. Density differences greater than threefold between the 72-h RANKL prime and $P$. aeruginosa LPS-mediated osteoclastogenesis groups are reported.

\section{Real-time quantitative RT-PCR analysis}

Total cellular RNA was extracted from RAW 264.7 cells with the use of Trizol $^{\circledR}$ reagent (Invitrogen) and further treated with DNaseI (Roche Applied Science). Reverse transcription of total RNA into cDNA was carried out with the Thermoscript ${ }^{\mathrm{TM}}$ RT-PCR System (Invitrogen) according to the manufacturer's instructions. Real-time polymerase chain reaction (PCR) 
amplification was performed using the SYBR Green PCR master mix kit (Applied Biosystems) in a $25 \mu \mathrm{l}$ reaction containing $0.9 \mu \mathrm{M}$ of each primer and $10 \mathrm{ng}$ of cDNA. Primers used were designed with Primer3 software (Rozen and Skaletsky 2000). Primer sequences were as follows (forward/reverse): $18 S$ (5'TCGGAACTGAGGC CATGAT-3' $/ 5^{\prime}$-TTTCGCTCTG GTCCGTCTTG-3'; accession no. X00686); IL-1R1 (5'CACTTC CCGTGCCTATGATT-3' / $5^{\prime}$-GGTTGGGATC TTAGCGTTGA-3'; accession no. NM_008362); NFATc 1 (5'-ACATAGCCTCCTGCTGGAAA-3' $/ 5^{\prime}$ AAGAGGGGTCTGGAGCAAAT- $3^{\prime}$; accession no. NM_016791); TNFR1 (5'-AGCCA CACCCACAACC TTAG-3' $/ 5^{\prime}$-TTTCACCCAC AGGGAGTAGG-3'; accession no. NM_011609); TNFR2 (5'-TGTAGAGGG GAAGGAACACG-3' / 5'-CACACCCAGGAACAGTC CTT-3'; accession no. NM_011610). DNA amplification and detection were carried out with the GeneAmp ${ }^{\circledR} 5700$ sequence detection system (Applied Biosystems). Thermal cycling conditions were as follows: $50^{\circ} \mathrm{C}$ for $2 \mathrm{~min}, 95^{\circ} \mathrm{C}$ for $10 \mathrm{~min}, 40$ cycles of $95^{\circ} \mathrm{C}$ for $15 \mathrm{~s}$, and $60-62^{\circ} \mathrm{C}$ for $1 \mathrm{~min}$. Following amplification, amplicon size and reaction specificity were confirmed by agarose gel electrophoresis and melting curve analysis. The cumulative fluorescence for each amplicon was normalized to that seen with 18 S ribosomal RNA amplification using the standard curve method (Livak 1997). Results were expressed as the fold increase (at each time point) over the respective $18 \mathrm{~S}$ controls. Means \pm SDs were calculated from a minimum of two separate experiments that were performed in triplicate.

\section{Statistical analysis}

All cell culture experiments were performed individually in triplicate and repeated a minimum of three times. Statistical significance across repeated experiments was evaluated using standard parametric statistics [i.e., paired $t$ test, one-way analysis of variance (ANOVA)]. An $\alpha$ level of 0.05 was established for significance. Significance across multiple groups after ANOVA analysis was tested with the Tukey test or the Bonferroni test where appropriate. All statistical calculations were performed with SigmaStat software (version 3.5, Systat Software, Richmond, CA, USA).

\section{RESULTS}

\section{P. aeruginosa LPS-mediated osteoclast formation in monocultures of $p O c s$}

As expected, RANKL priming alone without LPS at any time point was insufficient in stimulating Oc formation (Fig. 1A, two right wells). As demonstrated previously, no Ocs were seen if $P$. aeruginosa LPS was plated simultaneously with pOcs (Fig. 1A-C, zero hour time point; Zhuang et al. 2007). As soon as $24 \mathrm{~h}$ of RANKL priming, however, dose-dependent increases in Oc formation were observed with increased duration of RANKL priming $(p<0.01$, Fig. 1B). This increase in Oc formation was RANKLindependent since media containing LPS and without RANKL was sufficient in promoting osteoclastogenesis (Fig. 1B, white bars). Not surprisingly, the presence of RANKL always led to significantly more Ocs $(p<0.01$, Fig. 1B, black versus white bars). Importantly, Ocs formed in these experiments were capable of resorbing both dentin and hydroxyapatite (Fig. 1D).

Results obtained from primary pOcs were corroborated in RAW 264.7 cells, a murine hematopoietic cell line (Fig. 1C). Note that Oc formation for RAW cells is represented here by area fraction rather than total Oc number. Primed RAW cells are induced to fuse into syncitia to a greater degree than primary pOcs cells do, such that individual Ocs cannot be delineated or counted; therefore, Oc formation for RAW cells is represented here by area fractions rather than total Oc number.

\section{Cytokine profile of pOcs undergoing P. aeruginosa LPS-mediated osteoclast formation}

The finding of robust $P$. aeruginosa LPS-mediated Oc formation from RANKL-primed pOc monocultures suggested that Oc formation was the result of an LPSinduced autocrine/paracrine mechanism. In order to identify which cytokines were involved in this process, protein arrays were used to probe pure populations of RAW 264.7 cells exposed to different cell culture conditions (Fig. 2A). Untreated RAW cells in serum-containing media served as the baseline control (Fig. 2B). After correction for baseline cytokine concentrations, 11 cytokines were identified (Fig. 2E) at concentrations threefold or greater in pOcs undergoing LPS-mediated Oc formation (Fig. 2D) compared to pOcs primed with RANKL for $72 \mathrm{~h}$ (Fig. 2C).

In addition to TNF- $\alpha$ (Azuma et al. 2000), IL-1 $\alpha$ (Jimi et al. 1999), IL-6 (Kudo et al. 2003), monocyte chemoattractant protein-1 (MCP-1; Lu et al. 2007), macrophage inflammatory protein-1 alpha (MIP-1 $\alpha$; Lee et al. 2007), and granulocyte colony-stimulating factor (G-CSF; Hirbe et al. 2007), which have all recently been shown to promote Oc formation, we have also identified IL-10, IL-12p70, IL-9, leptin, and Regulated upon Activation, Normal T cell expressed, and secreted (RANTES) as highly expressed cytokines in pOcs undergoing $P$. aeruginosa LPS-mediated Oc formation. 


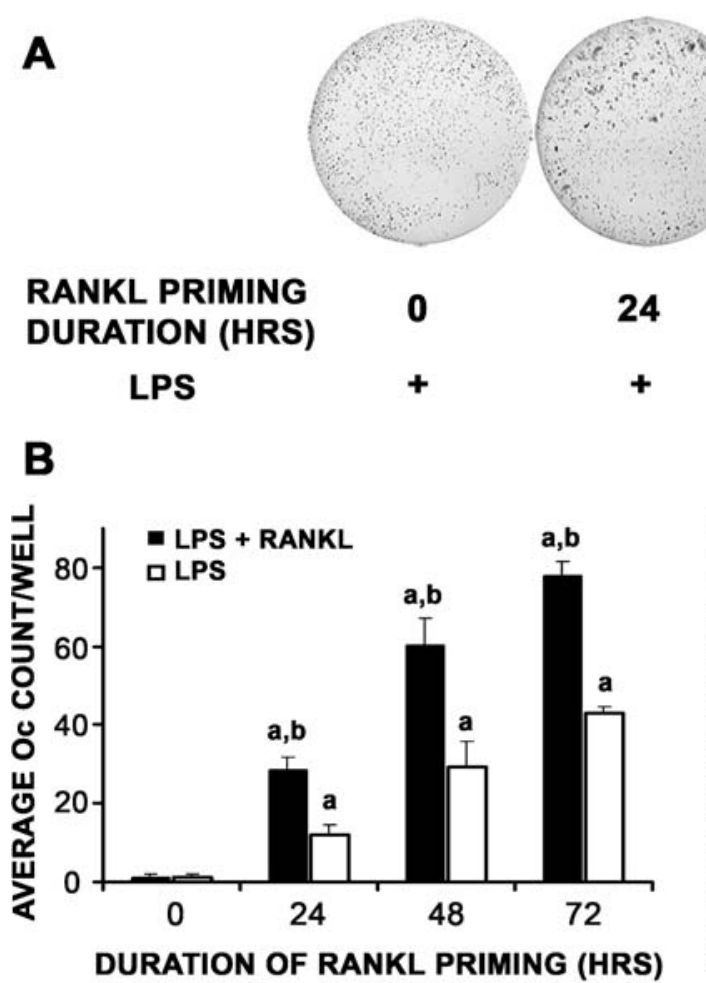

FIG. 1. Time course study of duration of RANKL priming on $P$. aeruginosa LPS-mediated Oc differentiation of primary pOcs. pOcs derived from bone marrow cells of $\mathrm{C} 57 \mathrm{BL} / 6$ mice were influenced to undergo LPS-mediated Oc formation with media containing LPS and M-CSF with/without RANKL. LPS-mediated Oc formation refers to RANKL (10 ng/ml) priming of pOcs for specified durations prior to $P$. aeruginosa LPS exposure. A Increases in Oc number were observed with increased duration of RANKL priming. Wells shown here received media containing LPS, M-CSF, and permissive RANKL after priming. B Dose-dependent increases in Oc number were observed with increased duration of RANKL priming in both treatment groups ( ${ }^{\mathrm{a}} p<0.01$, black and white bars). Furthermore, in groups undergoing 24,48 , and $72 \mathrm{~h}$ of RANKL priming, Oc formation was always significantly higher in those groups where RANKL was continuously present throughout the experiment (black versus white bars, ${ }^{\mathrm{b}} p<$ 0.01). C Time course study of duration of RANKL priming on $P$. aeruginosa LPS-mediated Oc differentiation of RAW 264.7 cells. RAW cells were influenced to undergo LPS-mediated Oc formation (as above) with media containing LPS with/without RANKL. Dosedependent increases in Oc area fraction were observed with increased duration of RANKL priming in both treatment groups $\left({ }^{a} p<0.01\right)$. D Representative photographs of resorption areas formed by primary pOcs on dentin (upper) and hydroxyapatite (lower) during $P$. aeruginosa LPS-mediated Oc formation. All data are expressed as the mean \pm SEM of at least three experiments performed in triplicate wells.
Cytokine involvement in P. aeruginosa LPS-mediated Oc formation

Using cytokine antibody arrays, we have identified many cytokines (Fig. 2E) that appear to be involved in $P$. aeruginosa LPS-mediated Oc formation. The roles of several of these inflammatory cytokines were further evaluated in our LPS-mediated osteoclastogenesis model by replacing wild-type (WT) primary pOcs with pOcs that were either incapable of releasing IL-6 $\left(\right.$ IL- $6^{-/-}$) or missing one or both TNF receptors $\left(\mathrm{TNFR} 1^{-/-}, \mathrm{TNFR} 2^{-/-}, \mathrm{TNFR} 1 / 2^{-/-}\right)$. Initially, these precursors with targeted deletions were compared to wild-type pOcs in their ability to undergo RANKLmediated Oc formation. Exposure to an osteoclastogenic dose of RANKL (100 $\mathrm{ng} / \mathrm{ml})$ revealed no differences between the pOcs in ability to undergo
Oc formation (Fig. 3, overall $p=0.105$ by one-way ANOVA with post hoc Bonferroni test).

IL- $6^{-/-}, \mathrm{TNFR} 1^{-/-}, \mathrm{TNFR} 2^{-/-}, \mathrm{TNFR} 1 / 2^{-/-}$, and IL-1R1 ${ }^{-/-}$pOcs were primed with RANKL for $72 \mathrm{~h}$ before stimulation with $P$. aeruginosa LPS and all demonstrated significant impairment in ability to undergo $P$. aeruginosa LPS-mediated Oc formation $(p<0.01$, Figs. $4 \mathrm{~A}-\mathrm{C})$. Involvement of IL-1 was further assessed in WT pOcs exposed concomitantly to IL-1 receptor antagonist (IL-1ra). As expected, addition of IL-1ra to WT pOcs progressing through $P$. aeruginosa LPS-mediated Oc formation also significantly inhibited osteoclastogenesis in a dose-dependent manner $(p<0.01$, Fig. 4D). Furthermore, as seen in Fig. 4B (hatched column), pOcs lacking both TNF receptors (TNR $1 / 2^{-/-}$) revealed almost no Oc formation and significantly more impairment than pOcs 
A. ARRAY LAYOUT

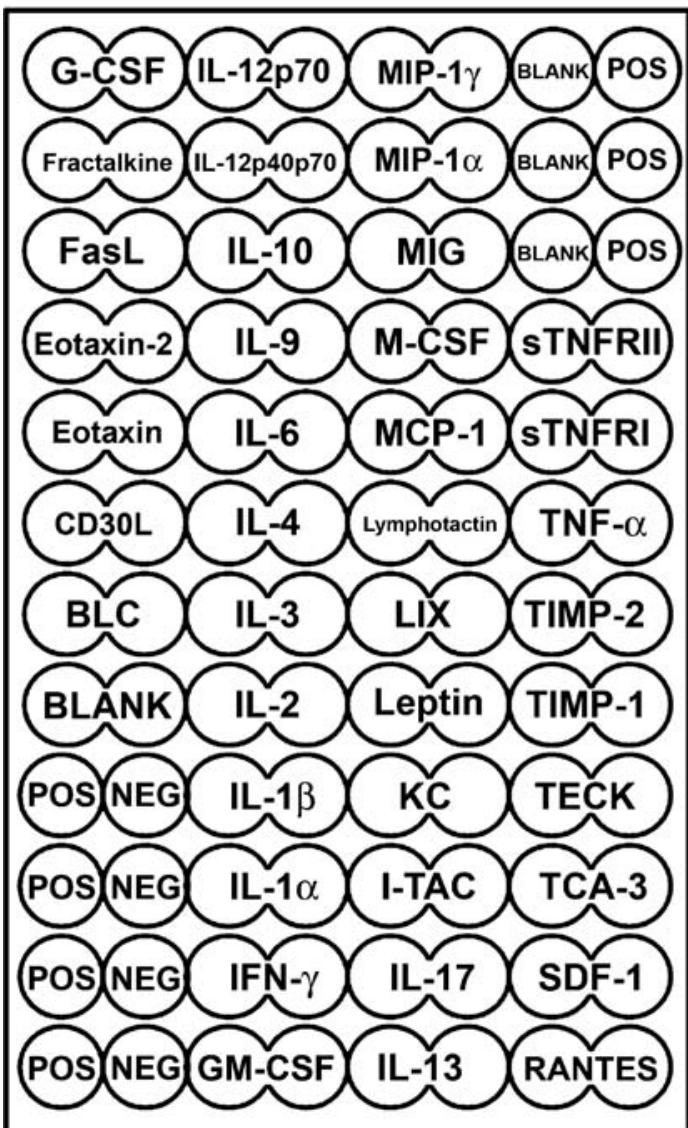

FIG. 2. Cytokine profile of pOcs undergoing RANKL priming and $P$. aeruginosa LPS-mediated osteoclastogenesis. A Cell culture supernatants were probed for the cytokines shown in the cytokine antibody array shown. B-D Array images represent one of two independent experiments that found similar patterns of expression. Untreated RAW cells (B), RAW cells primed with RANKL for $72 \mathrm{~h}(\mathbf{C})$, and RAW cells undergoing $P$. aeruginosa LPS-mediated Oc formation (D) were compared. In this case, $P$. aeruginosa LPS-mediated Oc formation refers to RAW cells primed for $72 \mathrm{~h}$ with RANKL $(10 \mathrm{ng} / \mathrm{ml}$ )

with single disruptions of either TNFR1 or TNFR2 (overall $p<0.001$, main $p<0.005$, Fig. 4B, hatched column). This suggested that signaling via both TNF receptor subtypes is required for optimal $P$. aeruginosa LPS-mediated osteoclastogenesis.

\section{Real-time quantitative PCR}

Ocs formed from $P$. aeruginosa LPS stimulation were also subjected to real-time quantitative PCR analysis. As Oc formation progressed, expression of nuclear factor of activated T cells c1 (NFATc1), the transcription factor identified as the "master switch regulator" of Oc formation (Takayanagi 2005), was twofold greater in pOcs progressing through $P$. aeruginosa LPS-mediated Oc formation as compared to pOcs

\section{72 HR RANKL} PRIME

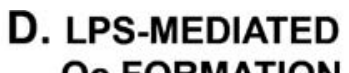

Oc FORMATION

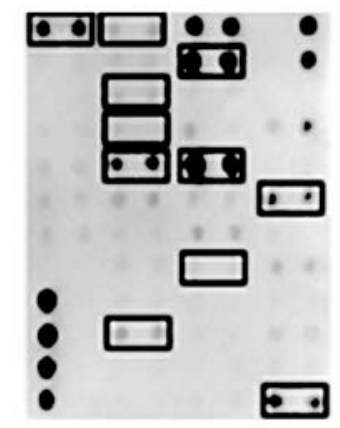

\section{E. RESULTS}

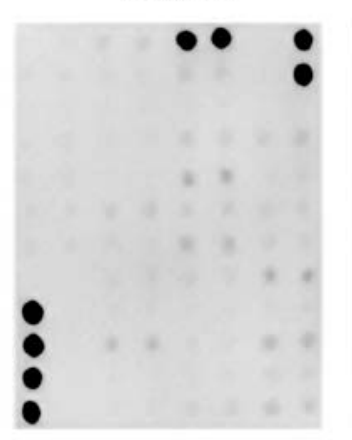

\begin{tabular}{|c|c|}
\hline & Expression Ratio \\
\hline & 180.9 \\
\hline & 101.2 \\
\hline & 56.4 \\
\hline & 12.2 \\
\hline & 10.1 \\
\hline & 5.6 \\
\hline & 4.4 \\
\hline & 4.4 \\
\hline & 4.0 \\
\hline & 3.5 \\
\hline
\end{tabular}

followed by $P$. aeruginosa LPS stimulation for 48 additional hours. D Cytokine ratios were calculated between the $P$. aeruginosa LPSmediated osteoclastogenesis and $72 \mathrm{~h}$ RANKL prime groups. Untreated RAW cell culture supernatants were used as the baseline control. Results presented are those cytokines whose mean cytokine concentrations were threefold or greater in pOcs undergoing LPS mediated Oc formation compared to those receiving only RANKL priming (highlighted in black boxes).

receiving only RANKL-priming ( $p<0.01$, Fig. 5 , white columns). This result provides transcriptional evidence that $P$. aeruginosa LPS stimulation of RANKLprimed pOcs drives these precursors towards Oc development.

In addition to NFATc1, expression of TNFR1 and TNFR2 were 2.5- and 21-fold greater in pOcs undergoing $96 \mathrm{~h}$ of LPS-mediated Oc formation as compared to pOcs receiving only RANKL priming $(p<$ 0.01 , Fig. 5, black/gray columns). Although expression of both receptors increased over time, TNFR2 expression increased significantly sooner than TNFR1. At $24 \mathrm{~h}$ of Oc development, TNFR2 transcription was 16 times higher in those pOcs progressing through LPS-mediated Oc formation as compared to RANKL-primed pOcs $(p<0.01$, Fig. 5, gray col- 


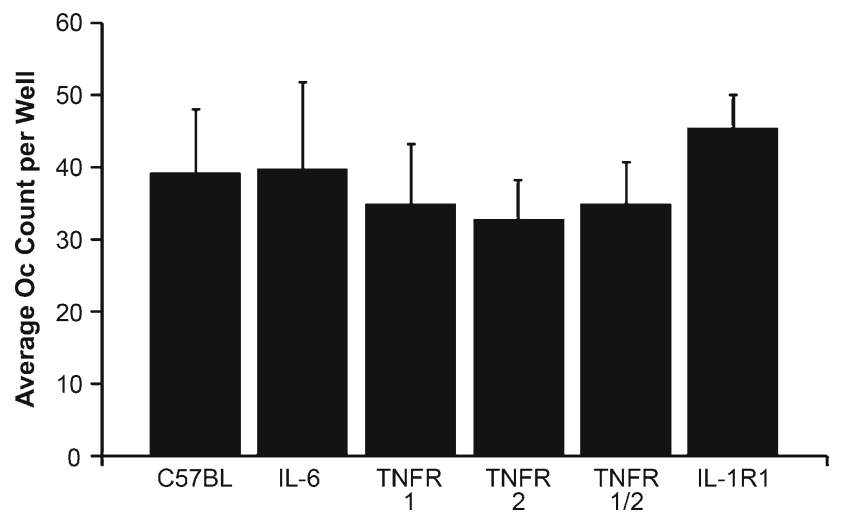

FIG. 3. RANKL-mediated osteoclastogenesis in wild-type and various knockout pOcs. Wild-type pOcs were compared to pOcs with targeted deletions of either IL-6 $\left(\mathrm{IL}-6^{-1-}\right)$, TNF receptors $\left(\mathrm{TNFR}^{-1-}{ }^{-1} \mathrm{TNFR}_{2}^{--}\right.$, $\mathrm{TNFR}_{1 / 2^{-/-}}$), or IL-1 receptor $1\left(\mathrm{IL}-1 \mathrm{R} 1^{-1-}\right.$ ) in their ability to form Ocs by exposing them to RANKL at $100 \mathrm{ng} / \mathrm{ml}$ for 6 days in the presence of M-CSF $(10 \mathrm{ng} / \mathrm{ml})$. There were no differences in the abilities of any of these cell types to form Ocs under these conditions (overall $p=0.105$ by one-way ANOVA with post hoc Bonferroni test). All data are expressed as the mean \pm SD of at least three experiments performed in triplicate wells.

umns). Clearly, TNFR1 expression was not significant at this time point and never reached the same level of expression as TNFR2 (21-fold versus 2.5-fold).

Expression of IL-1R1 was similarly increased in pOcs as they transformed into Ocs under the influence of $P$. aeruginosa LPS. Although IL-1R1 expression was not significantly increased in pOcs progressing through $24 \mathrm{~h}$ of osteoclastogenesis, expression at $96 \mathrm{~h}$ of osteoclastogenesis was 190 times greater in pOcs undergoing $P$. aeruginosa LPS-mediated osteoclastogenesis as compared to RANKL-primed pOcs $(p<0.01$, Fig. 5, striped column).

FIG. 4. Autocrine IL-6, TNF- $\alpha$, and IL-1 involvement in LPSmediated osteoclastogenesis. A-C pOcs derived from mice incapable of generating IL-6 (IL-6 $\left.{ }^{--}\right)$, mice lacking one or both TNF receptors (TNFR1 $1^{-/-}, \mathrm{TNFR}_{2}{ }^{--}$, or TNFR $1 / 2^{-/-}$), or mice incapable of responding to $\mathrm{IL}-1\left(\mathrm{IL}-1 \mathrm{R} 1^{-1}\right)$ were influenced to undergo $P$. aeruginosa LPS-mediated Oc formation. In this case, $P$. aeruginosa LPS-mediated Oc formation refers to $72 \mathrm{~h}$ of RANKL $(10 \mathrm{ng} / \mathrm{ml})$ priming followed by $P$. aeruginosa LPS stimulation for an additional 96 h. Clearly, IL-6 ${ }^{--}$pOcs (A), all TNF receptor knockout subtype pOcs $(\mathbf{B})$, and IL-1R $1^{-/-}$pOcs $(\mathbf{C})$ were impaired in their ability to undergo LPS-mediated Oc formation when compared to $\mathrm{C} 57 \mathrm{BL} / 6$ wild-type control pOcs $\left({ }^{\mathrm{a}} p<0.01\right)$. Furthermore, TNFR $1 / 2^{-/-}$double knockout pOcs demonstrated significantly more impairment than either TNFR1 ${ }^{-/-}$or TNFR2 ${ }^{-/-}$pOcs (overall $p<0.001$, ${ }^{\text {b }}$ main $p<0.005$ by one-way ANOVA with post hoc Tukey test). D. C57BL/6 wild-type pOcs undergoing $P$. aeruginosa LPS-mediated Oc formation were concomitantly exposed to IL-1 ra $(0,1,10,20 \mu \mathrm{g} / \mathrm{ml})$. Clearly, decreased Oc formation was seen with increasing IL-1 ra dose and achieved statistical significance at 10 and $20 \mu \mathrm{g} / \mathrm{ml}\left({ }^{\mathrm{a}} p<0.01\right)$. All data are expressed as the mean \pm SEM of at least three experiments performed in triplicate wells.

\section{DISCUSSION}

In this study, we have demonstrated that $P$. aeruginosa LPS is capable of stimulating the transformation of pOc monocultures into bone-resorbing Ocs (Fig. 1). Importantly, $P$. aeruginosa LPS-mediated osteoclastogenesis would only occur if pOcs were previously exposed to permissive levels of RANKL, a process
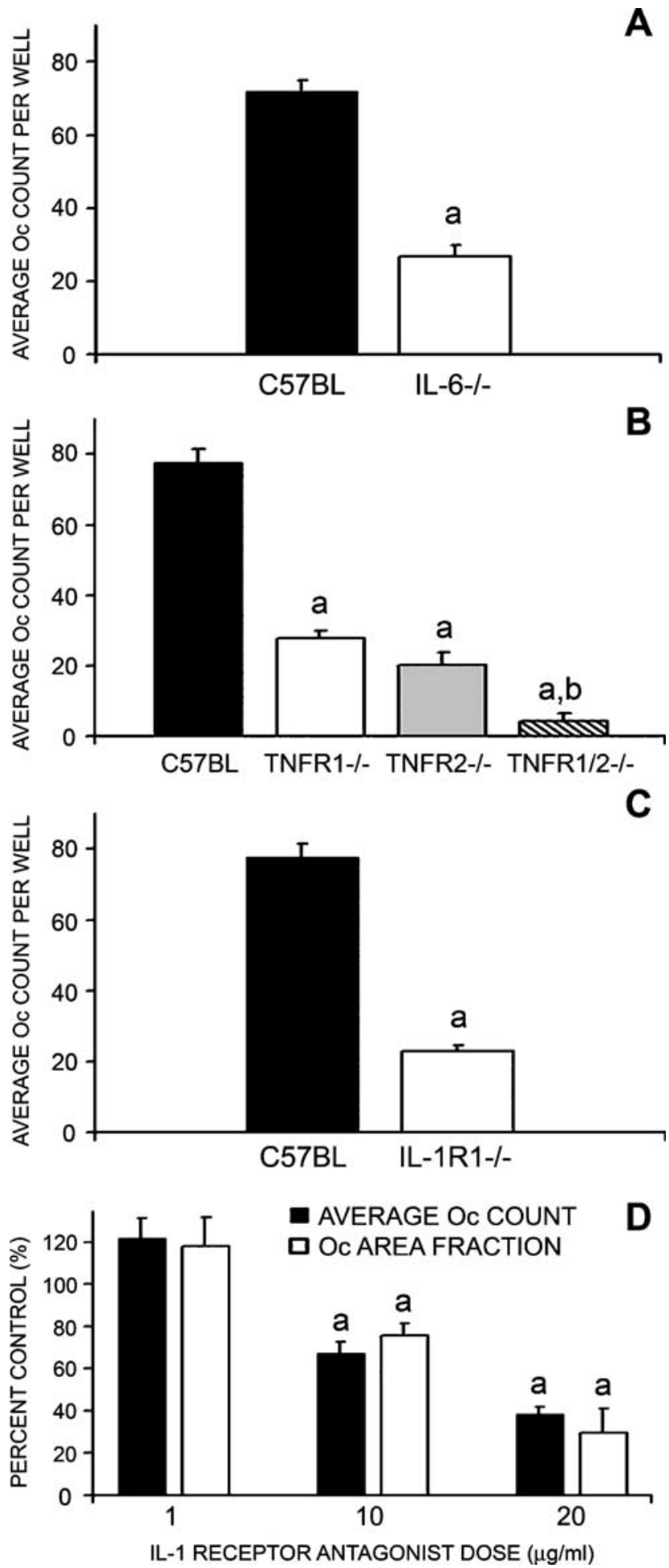


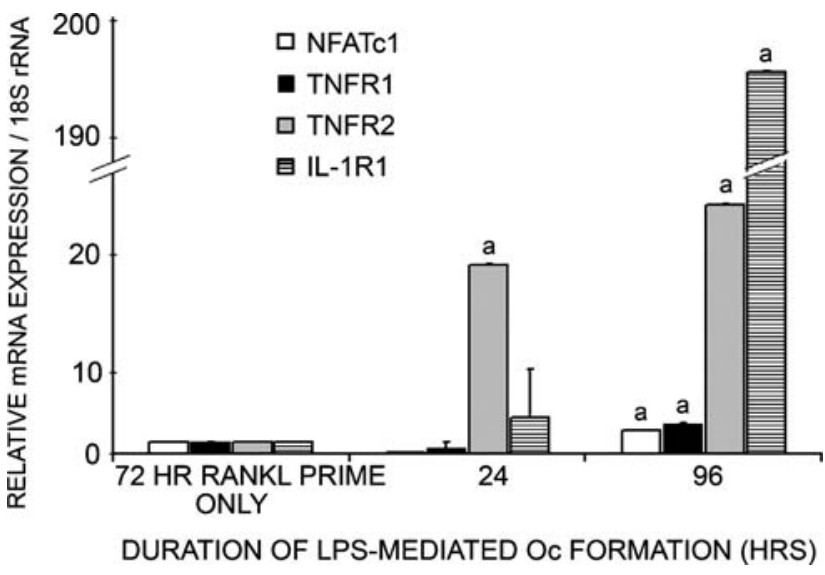

FIG. 5. Quantitative PCR analysis of TNFR1, TNFR2, IL-1R1, and NFATc1 expression by pOcs undergoing $P$. aeruginosa LPS-mediated Oc formation. Here, $P$. aeruginosa LPS-mediated Oc formation refers to $72 \mathrm{~h}$ of RANKL $(10 \mathrm{ng} / \mathrm{ml})$ priming followed by P. aeruginosa LPS stimulation for an additional $96 \mathrm{~h}$. RNA was harvested for PCR analysis at the following time points: (1) after $72 \mathrm{~h}$ of RANKL priming; (2) $24 \mathrm{~h}$ after $P$. aeruginosa LPS addition; and (3) $96 \mathrm{~h}$ after $P$. aeruginosa LPS addition. RAW 264.7 pOcs undergoing $P$. aeruginosa LPS-mediated Oc formation clearly show significantly increased expression of IL-1R1, TNFR1, TNFR2, and NFATC1 as maturation into Ocs progresses $\left({ }^{\mathrm{a}} p<0.01\right)$. The data are expressed as the mean $\pm \mathrm{SD}$ of at least two experiments performed in triplicate.

termed "RANKL priming" (Lam et al. 2000; Zou and Bar-Shavit 2002). RANKL priming doses do not induce osteoclastogenesis alone (Fig. 1A) and are deemed "permissive" in that multinucleation and Oc formation may occur with downstream stimuli.

Although our results are consistent with several studies, others have demonstrated that LPS antagonizes osteoclastogenesis. In the study by Takami et al. (2002) for example, bone marrow monocytes exposed to Escherichia coli LPS did not develop into Ocs; rather, these LPS-exposed pOcs retained phagocytic ability, an immune function typical of a macrophage. Although macrophages and Ocs share a common monocyte precursor, maturing pOcs lose both phagocytic ability and capacity for inflammatory cytokine secretion as they develop into Ocs (Itoh et al. 2003). Importantly, marrow monocytes in the above study were not initially primed with RANKL, and this omission clearly caused them to develop into a nonosteoclastic, immune cell type upon LPS exposure. We have obtained similar results when pOcs were not initially RANKL primed (Zhuang et al. 2007). Taken together, these studies demonstrate that although RANKL priming does not induce osteoclastogenesis by itself, priming undoubtedly directs monocytes towards the Oc lineage. Our results have shown that exposure to $P$. aeruginosa LPS after a RANKL priming period leads to continued differentiation towards the Oc phenotype.
The finding of robust $P$. aeruginosa LPS-mediated osteoclastogenesis in a pure population of RANKLprimed RAW 264.7 cells suggests that LPS stimulates RANKL-primed pOcs to release pro-osteoclastogenic factors in an autocrine/paracrine manner. Importantly, this autocrine/paracrine mechanism is sufficient to sustain Oc formation even after RANKL withdrawal (Fig. 1B, white bars). Once sufficient priming has occurred, these results suggest that proosteoclastogenic cytokines other than RANKL are elaborated upon LPS exposure.

Using cytokine antibody arrays, we have identified 11 cytokines that are expressed in significantly increased concentrations in pOcs undergoing $P$. aeruginosa LPS-mediated osteoclastogenesis (Fig. 2E). LPS is well known to elicit TNF- $\alpha$ (Abu-Amer et al. 1997; Zou and Bar-Shavit 2002; Itoh et al. 2003), IL-6 (Itoh et al. 2003; Sato et al. 2004), IL-1 $\alpha$ (Hong et al. 2004), and IL-1 $\beta$ (Itoh et al. 2003) release from monocytes. It is also well established that these cytokines promote Oc formation (Jimi et al. 1999; Azuma et al. 2000; Zhang et al. 2001; Zou et al. 2001; Kudo et al. 2003; Wei et al. 2005). We have confirmed their pro-osteoclastogenic properties but also shown for the first time that these cytokines are released by RANKL-primed pOcs autonomously when exposed to LPS (Fig. 2).

In addition to identifying the autocrine proosteoclastogenic involvement of TNF- $\alpha$ in $P$. aeruginosa LPS-mediated osteoclastogenesis, we have identified that both TNF receptor subtypes, TNFR1 (p55) and TNFR2 (p75), are also needed for optimal Oc formation. Absence of both receptor subtypes virtually abrogated any osteoclastogenesis (Fig. 4B). Interestingly, expression of both of these receptors is significantly increased in pOcs as they progress through Oc maturation in our inflammatory model (Fig. 5). TNFR2 expression increases significantly before TNFR1 expression, suggesting that TNFR2 may play a more pivotal role than TNFR1 in early inflammatory Oc formation. Taken together, these findings suggest that one mechanism underlying autonomous $P$. aeruginosa LPS-mediated Oc formation is increased expression of TNF- $\alpha$, TNFR1, and TNFR2, all of which promote osteoclastogenesis.

Increased expression of both receptors would sensitize pOcs to TNF- $\alpha$ in the microenvironment and strengthen a pro-osteoclastogenic autocrine feedback loop.

The finding of TNFR2-dependence in our study contrasts in vivo data using $\mathrm{C} 3 \mathrm{H} / \mathrm{HeN}$ mice that demonstrated dependence only on TNFR1 (AbuAmer et al. 1997). It also contrasts an in vitro study using Balb/c and C57 mice that revealed TNFR1 but not TNFR2 dependence (Zou et al. 2001). Interestingly, the latter study demonstrated that LPS induced release of TNF- $\alpha$ which then promoted Oc formation 
by an autocrine mechanism. However, Oc formation in this study occurred only in Balb/c and not C57BL/ 6 mice, the animals used in our study. Previous studies have demonstrated that $\mathrm{Balb} / \mathrm{c}$ and $\mathrm{C} 57 \mathrm{BL} / 6$ mice mount different responses to inflammatory stimuli (Sapru et al. 1999). Such differences likely contributed to these conflicting results.

We have also shown that $P$. aeruginosa LPS-mediated Oc formation involves greatly increased pOc expression of IL-1R1 as maturation into Ocs progresses (Fig. 5). Such a mechanism would also sensitize the pOc to IL-1, a pro-osteoclastogenic cytokine released in high concentrations by pOcs during $P$. aeruginosa LPS-mediated osteoclastogenesis (Fig. 2E). It is interesting to note that increased IL-1R1 expression occurs well after IL-1 $\alpha$ and IL-1 $\beta$ expression (within $24 \mathrm{~h}$ of LPS stimulation, R. Nason, unpublished data). These time course data suggest that IL-1 release may cause upregulation of IL-1R1 and the establishment of another autocrine loop. Alternatively, IL-1R1 and IL1 upregulation may be due to pOc stimulation by TNF- $\alpha$ or LPS. This particular sequence of events is currently being investigated in our laboratory.

Our study has also identified G-CSF, IL-10, IL12p70, MCP-1, IL-9, MIP-1 $\alpha$, leptin, and RANTES as cytokines secreted by pOcs undergoing $P$. aeruginosa LPS-mediated osteoclastogenesis. Of note, G-CSF (Hirbe et al. 2007), MCP-1 (Lu et al. 2007), and MIP-1 $\alpha$ (Lee et al. 2007), have recently been shown to promote Oc formation. Interestingly, IL-10 (Owens et al. 1996), IL-12 (Horwood et al. 2001), and leptin (Cornish et al. 2002) have all been shown to possess anti-osteoclastogenic activities. Since pOcs release numerous cytokines, many of which are redundant in their ability to promote or antagonize Oc formation, it is likely that Oc formation from RANKL-primed pOcs occurs in response to LPS if the overall balance of LPS-elicited cytokines favors osteoclastogenesis.

In this study, we have taken a different approach in studying infection-related inflammatory osteolysis. By eliminating the presence of supporting cells (i.e., Obs, lymphocytes) capable of cytokine production, we have established a model of robust osteoclastogenesis induced by $P$. aeruginosa LPS in pure populations of RANKL-primed pOcs. Importantly, priming involves a permissive dose of RANKL in the pOc microenvironment; such a dose does not induce multinucleation and Oc formation but clearly directs mononuclear precursors towards the Oc lineage. Inflammatory conditions like COM and infected cholesteatomas in which elevated concentrations of RANKL (Gravallese et al. 2000; Hofbauer and Heufelder 2001) and LPS (Peek et al. 2003) have been demonstrated represent microenvironments which could theoretically support this autonomous mode of Oc activation. In such a setting, priming by local RANKL with subsequent activation by pervasive LPS would lead to robust osteoclastogenesis.

We have discovered that RANKL-primed pOcs are fully capable of transforming themselves into boneresorbing Ocs once stimulated with $P$. aeruginosa LPS. After sufficient priming with sub-osteoclastogenic levels of RANKL, we have shown that $P$. aeruginosa LPS stimulates pOc release of TNF- $\alpha$, IL- $1 \alpha$, and IL- 6 , all of which subsequently promote Oc formation in an autocrine/paracrine manner. This autonomous mode of Oc formation is further supported by concomitantly increased expression of TNFR1, TNFR2, and IL-1R1, establishing possibly increased pOc responsiveness to TNF- $\alpha$ and IL- $1 \alpha$. The ability of pOcs to respond to LPS by autonomously generating a host of proosteoclastogenic cytokines suggests that these cells may play a greater role in the propagation of pathologic bone resorption. Therefore, it is interesting to speculate that this mechanism may also be involved in the formation of multinucleated giant cells seen in other chronic inflammatory states. Although the role of RANKL priming in osteoclastogenesis in the context of this work is independent of LPS type, it must also be appreciated that LPS molecules from different $P$. aeruginosa serotypes as well as other Gram-negative species may induce different in vivo and vitro effects. Since chronic otitis may harbor many Gram-negative species, we are also currently pursuing the differential effects of different LPS molecules in our model.

\section{ACKNOWLEDGMENTS}

This work was supported by NIH grants R01-DC000263-21 and P30-DC004665-07 to R.A.C. from the National Institute on Deafness and Other Communicative Disorders (National Institutes of Health, Bethesda, MD). R.N. is also supported by NIH training grant T32 DC00022. We would like to thank Mary-Pashia Basse for her technical assistance with real-time PCR experiments.

\section{REFERENCES}

Abu-Amer Y, Ross FP, eT AL. Lipopolysaccharide-stimulated osteoclastogenesis is mediated by tumor necrosis factor via its P55 receptor. J. Clin. Invest. 100(6):1557-1565, 1997.

Alliston T, Derinck R. Medicine: interfering with bone remodelling. Nature 416(6882):686-687, 2002.

Azuma Y, Kaji K, ET AL. Tumor necrosis factor-alpha induces differentiation of and bone resorption by osteoclasts. J. Biol. Chem. 275(7):4858-4864, 2000.

Cornish J, Callon KE, ET AL. Leptin directly regulates bone cell function in vitro and reduces bone fragility in vivo. J. Endocrinol. 175(2):405-415, 2002.

ERICKSON SL, DE SAUVAgE FJ, ET AL. Decreased sensitivity to tumournecrosis factor but normal T-cell development in TNF receptor2-deficient mice. Nature 372(6506):560-563, 1994. 
Felix R, Cecchini MG, et AL. Impairment of macrophage colonystimulating factor production and lack of resident bone marrow macrophages in the osteopetrotic op/op mouse. J. Bone Miner. Res. 5(7):781-789, 1990.

Fuiss DM, Sнонам I, ET AL. Chronic suppurative otitis media without cholesteatoma in children in southern Israel: incidence and risk factors. Pediatr. Infect. Dis. J. 10(12):895-899, 1991.

Glaccum MB, Stocking KL, et al. Phenotypic and functional characterization of mice that lack the type I receptor for IL-1. J. Immunol. 159(7):3364-3371, 1997.

Gravallese EM, Manning C, Et al. Synovial tissue in rheumatoid arthritis is a source of osteoclast differentiation factor. Arthritis Rheum. 43(2):250-258, 2000.

Hirbe AC, Uluckan O, et al. Granulocyte colony-stimulating factor enhances bone tumor growth in mice in an osteoclast-dependent manner. Blood 109(8):3424-3431, 2007.

Hofbauer LC, Heufelder AE. Role of receptor activator of nuclear factor-kappaB ligand and osteoprotegerin in bone cell biology. J. Mol. Med. 79(5-6):243-253, 2001.

Hong CY, LIN SK, ET AL. The role of lipopolysaccharide in infectious bone resorption of periapical lesion. J. Oral. Pathol. Med. 33(3):162-169, 2004.

Horwood NJ, Elliott J, ET AL. IL-12 alone and in synergy with IL-18 inhibits osteoclast formation in vitro. J. Immunol. 166(8):49154921, 2001.

Hsu H, Lacey DL, ET al. Tumor necrosis factor receptor family member RANK mediates osteoclast differentiation and activation induced by osteoprotegerin ligand. Proc. Natl. Acad. Sci. U. S. A. 96(7):3540-3545, 1999.

ITOH K, UdAGAWA N, ET AL. Lipopolysaccharide promotes the survival of osteoclasts via Toll-like receptor 4, but cytokine production of osteoclasts in response to lipopolysaccharide is different from that of macrophages. J. Immunol. 170(7):3688-3695, 2003.

Jimi E, NaKamura I, et AL. Interleukin 1 induces multinucleation and bone-resorbing activity of osteoclasts in the absence of osteoblasts/stromal cells. Exp. Cell Res. 247(1):84-93, 1999.

Kong YY, Feige U, et Al. Activated T cells regulate bone loss and joint destruction in adjuvant arthritis through osteoprotegerin ligand. Nature 402(6759):304-309, 1999.

Kopf M, Baumann H, et AL. Impaired immune and acute-phase responses in interleukin-6-deficient mice. Nature 368(6469):339-342, 1994.

Kudo O, Saвоквar A, et al. Interleukin-6 and interleukin-11 support human osteoclast formation by a RANKL-independent mechanism. Bone 32(1):1-7, 2003.

LACEY DL, Timms E, ET AL. Osteoprotegerin ligand is a cytokine that regulates osteoclast differentiation and activation. Cell 93(2): $165-176,1998$.

LAM J, TAKeShita S, ET AL. TNF-alpha induces osteoclastogenesis by direct stimulation of macrophages exposed to permissive levels of RANK ligand. J. Clin. Invest. 106(12):1481-1488, 2000.

LEe JE, Shin HH, ET AL. Stimulation of osteoclastogenesis by enhanced levels of MIP-1alpha in BALB/c mice in vitro. Exp. Hematol. 35(7):1100-1108, 2007.
Lrvak KJ. ABI Prism 7700 sequence detection system. User Bulletin no. 2. from http://docs.appliedbiosystems.com/pebiodocs/ 04303859.pdf, 1997.

LU Y, CAI Z, ET AL. Monocyte chemotactic protein-1 mediates prostate cancer-induced bone resorption. Cancer Res. 67(8):3646-3653, 2007.

Nakagana T, Yadohisa O, ET AL. A 16-year survey of changes in bacterial isolates associated with chronic suppurative otitis media. Eur. Arch. Otorhinolaryngol. 251(Suppl 1):S27-S32, 1994.

Owens JM, Gallagher AC, ET AL. IL-10 modulates formation of osteoclasts in murine hemopoietic cultures. J. Immunol. 157(2): 936-940, 1996.

Peek FA, Huisman MA, et Al. Lipopolysaccharide concentration and bone resorption in cholesteatoma. Otol. Neurotol. 24(5):709713, 2003.

Peschon JJ, Torrance DS, et al. TNF receptor-deficient mice reveal divergent roles for p55 and p75 in several models of inflammation. J. Immunol. 160(2):943-952, 1998.

RASBAND WS. (1997-2007). ImageJ. from http://rsb.info.nih.gov/ij/.

Rozen S, Skaletsky HJ. Primer3 on the WWW for general users and for biologist programmers. In: Krawetz S, Misener S (eds) Bioinformatics Methods and Protocols: Methods in Molecular Biology. Totowa, NJ, Humana, pp. 365-386, 2000.

SAPru K, Stotland PK, ET AL. Quantitative and qualitative differences in bronchoalveolar inflammatory cells in Pseudomonas aeruginosaresistant and -susceptible mice. Clin. Exp. Immunol. 115(1): 103-109, 1999.

Sato N, TAKahashi N, ET al. MyD88 but not TRIF is essential for osteoclastogenesis induced by lipopolysaccharide, diacyl lipopeptide, and IL-1alpha. J. Exp. Med. 200(5):601-611, 2004.

Tакамг M, Kim N, eт al. Stimulation by toll-like receptors inhibits osteoclast differentiation. J. Immunol. 169(3):1516-1523, 2002.

TAKAYANAGI H. Inflammatory bone destruction and osteoimmunology. J. Periodontal. Res. 40(4):287-293, 2005.

Udagawa N, TAKAHASHI N, ET AL. Origin of osteoclasts: mature monocytes and macrophages are capable of differentiating into osteoclasts under a suitable microenvironment prepared by bone marrow-derived stromal cells. Proc. Natl. Acad. Sci. U. S. A. 87(18):7260-7264, 1990.

Wei S, Kitaura H, et al. IL-1 mediates TNF-induced osteoclastogenesis. J. Clin. Invest. 115(2):282-290, 2005.

Zhang YH, Heulsmann A, ET AL. Tumor necrosis factor-alpha (TNF) stimulates RANKL-induced osteoclastogenesis via coupling of TNF type 1 receptor and RANK signaling pathways. J. Biol. Chem. 276(1):563-568, 2001.

Zhuang L, Jung JY, et al. Pseudomonas aeruginosa lipopolysaccharide induces osteoclastogenesis through a toll-like receptor 4 mediated pathway in vitro and in vivo. Laryngoscope 117(5):841-847, 2007.

Zou W, Bar-Shavit Z. Dual modulation of osteoclast differentiation by lipopolysaccharide. J. Bone Miner. Res. 17(7):1211-1218, 2002.

Zou W, HaKiм I, et AL. Tumor necrosis factor-alpha mediates RANK ligand stimulation of osteoclast differentiation by an autocrine mechanism. J. Cell. Biochem. 83(1):70-83, 2001. 